\title{
Identification of a nonsense mutation in TNNI3K associated with cardiac conduction disease and dilated cardiomyopathy
}

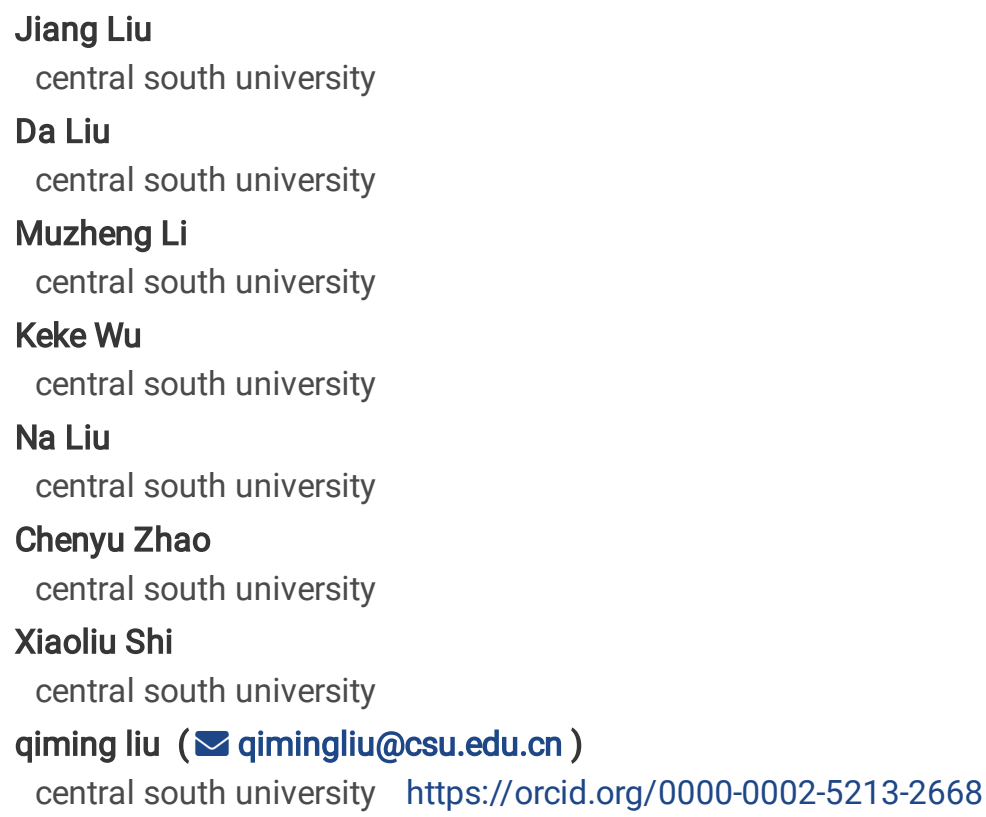

\section{Research article}

Keywords: conduction disease;dilated cardiomyopathy;nonsense mutation;TNNI3K

Posted Date: December 9th, 2019

DOl: https://doi.org/10.21203/rs.2.18398/v1

License: (c) (i) This work is licensed under a Creative Commons Attribution 4.0 International License. Read Full License 


\section{Abstract}

Background Cardiac conduction disease(CCD) and dilated cardiomyopathy (DCM) are two common cardiovascular diseases which can lead to life-threatening conditions. The importance of heredity in these two diseases has been realized in recent years. Several casual genes have been found to be implicated in CCD and DCM such as LMNA,SCN5A,TTN,TNNI3K and so on. Rare genetic mutations in TNNI3K have been identified to be in connection with CCD and DCM.Methods Next generation sequencing(NGS) was carried out in order to identify the underlying disease-causing mutation in a Chinese family with CCD and DCM. The mutations were validated by Sanger sequencing.Results A nonsense mutation in TNNI3K(NM_015978.2: g.170891C>T,C.1441C>T) was identified in this family and validated by Sanger sequencing.Conclusions This study described a Chinese family with CCD and DCM caused by a nonsense TNNI3K mutation. TNNI3K harboring the mutation(c.1441C $>\mathrm{T}$ ) possibly implicated a loss-of-function pathogenic mechanism with an autosomal dominant inheritance pattern. This research enriches the phenotypic spectrum of TNNI3K mutations, casting a new light upon the genotype-phenotype correlations between TNNI3K mutations and cardiovascular diseases.

\section{Background}

Cardiac conduction disease(CCD) refers to the impaired completeness of conduction system which can be serious and potentially life-threatening. Based on part upon the site of conduction block,CCD is recognized,namely,sick sinus syndrome, Intra-atrial block ,atrioventricular block and intraventricular conduction delay. More than 50 genes have been found to be relevant with CCD such as SCN5A,TRPM4,NKX2.5,TBX5,PRKAG2,and LMNA[1]. CCD continues to be the major cause for pacemaker(PM) implantation irrespective of its diverse underlying pathophysiological mechanisms[2, 3]. Dilated cardiomyopathy(DCM),characterized by ventricles dilation and impaired contraction without abnormal loading conditions or coronary artery disease sufficient to explain the abnormalities[4, 5],remains to be a principal cause of death worldwide. The prevalence of DCM was estimated to be 36 cases per 100,000 population[6]. Over 100 genes have been implicated in harboring rare variants that cause DCM,containing genes that encode cytoskeletal, sarcomere,nuclear envelope proteins and so on[7, 8]. Up to now there have been over 30 genes confirmed to lead to both CCD and DCM.

TNNI3K, located in chromosome 1(1p31.1),encodes a dual-function kinase(both tyrosine and serine/threonine kinase activity) with biased expression in heart(OMIM \#616117)[7, 8]. It contains four domains as follow:a N-terminal coiled coil domain,ankyrin(ANK) repeats,protein kinase domain and a C-terminal serine-rich domain. Interaction partners of TNNI3K embody cardiac troponin I(cTnI),anti-oxidant protein 1(AOP-1) and p38,which make TNNI3K an important factor in cardiovascular diseases[11, 12]. TNNI3K were related with heart failure and hypertrophy,ischemia/reperfusion injury, cardiac conduction,and heart regeneration[13-19]. To date there are only four mutations that have been found to be relevant with cardiovascular diseases,including three missense mutations and one splicing mutation[20-23]. Here,we found that a nonsense variant in TNNI3K(NM_015978.2:c.1441C > T) ,which was not seen in the human gene mutation database(HGMD), may be the cause of cardiac abnormalities.

\section{Methods}

\section{Patients and Ethics}

A Chinese family with cardiovascular diseases was enrolled in our studies. Parents of the proband are not related biologically. The pedigree of the family is shown in Fig. 1A.

\section{Next generation sequencing}

Whole exome sequencing(WES) was performed on the proband (III-10) in this family. The next generation sequencing(NGS) and basic bioinformatics analysis were finished in the Novogene Bioinformatics. Institute (Beijing, China). Platform for high-throughput sequencing was Illumina novaseq. The reads data were mapped to the human reference genome 19 (UCSC hg19) using BWA. Single-nucleotide variants (SNVs) and insertion-deletion variants (INDELs) were annotated by ANNOVAR. Eliminate low-quality data(cover depth $\$ 20 x$ ) and high-frequency mutation sites[minor allele frequency (MAF) >0.001 in ExAC,ESP6500,1000 Genome Project, and gnomAD]in general population from raw data.SIFT,PolyPhen-2,MutationTaster,and provean were used for predicting pathogenicity of mutations. Interpretation of mutations pathogenicity was guided by American College of Medical Genetics and Genomics guideline(ACMG)[24]. The filter strategies is shown in Fig. 1B and Table1. The validation of potential mutations from NGS 
was done by means of Sanger sequencing. Primer 3 was used to design the primer pairs(we'll offer the primers sequences upon request). ABI 3730 sequencer (Applied Biosystems, Foster City, CA, USA) was used for Sanger sequencing.

Table 1

The gene list of Sanger sequencing validation and co - segregation analysis in family - 2

\begin{tabular}{|llllllllll|}
\hline CHR & POS & RB & AB & Gene & AAChange & SIFT & $\begin{array}{l}\text { PolyPhen } \\
-2\end{array}$ & Mutationtaster \\
\hline 1 & 74834917 & C & T & TNNI3K & NM_015978:exon15:C.C1441T & - & - & $1.000, D$ \\
\hline 1 & 237947718 & T & G & RYR2 & NM_001035:exon90:C.T12706G & $0.5, T$ & $0.257, B$ & $1.000, D$ \\
\hline 2 & 179425543 & C & T & TTN & NM_001267550:exon326:C.G85316A & $0, D$ & $0.701, P$ & $1.000, D$ \\
\hline 12 & 2788847 & C & T & CACNA1C & NM_000719:exon42:c.C5329T & $0.002, D$ & $0.999, D$ & $1.000, D$ \\
\hline 14 & 32319403 & A & C & NUBPL & NM_001201573:exon8:C.A605C & $0.23, T$ & $0.002, B$ & $0.764, N$ \\
\hline 18 & 3164344 & C & A & MYOM1 & NM_003803:exon10:C.G1433T & $0.31, T$ & $1.000, D$ & $0.764, N$ \\
\hline
\end{tabular}

\section{Results}

\section{Clinical data}

The index case(III-10) is a 56-year-old man from China with the clinical manifestation of chest tightness. The electrocardiogram(ECG) showed complete right bundle branch block(CRBBB)and third-degree atrioventricular block(Fig. 1C). The 24hour holter indicated an average heat beat of $46 \mathrm{bpm}$, maximum RR interval of 5.26 seconds,complete atrioventricular block and CRBBB. His Echocardiography displayed enlargement of left atrial $(\mathrm{LA}=40 \mathrm{~mm})$, thickened left ventricular posterior wall (LVPW = $13 \mathrm{~mm}$ ) and interventricular septum (IVS = $14 \mathrm{~mm}$ )(Fig. 1D). His father and uncles(II-5,II-7,II-9 and II-11) were also suffered from third-degree atrioventricular block and were treated with pacemaker therapy among their $50 \mathrm{~s}$. Results of echocardiography were all normal before their pacemakers implantation. Cardiac enlargement of one of his uncles(II-7) emerged after a period of pacemaker implantation(left ventricle from $54 \mathrm{~mm}$ to $62 \mathrm{~mm}$,left atrial from $38 \mathrm{~mm}$ to $42 \mathrm{~mm}$,right ventricle from $28 \mathrm{~mm}$ to $39 \mathrm{~mm}$ and right atrial from $27 \mathrm{~mm}$ to $37 \mathrm{~mm}$ ). The proband's aunt(II-14) was died from heart disease at her $30 \mathrm{~s}$ recalled by him(no more details available). Owing to the implantation of PM,cardiac magnetic resonance is not performed in this family(Table 2). 
Table 2

Clinical Characteristics and Phenotype of Family Members

\begin{tabular}{|c|c|c|c|c|c|}
\hline Subject & Sex & $\begin{array}{l}\text { Age } \\
\text { (years/old) }\end{array}$ & $\begin{array}{l}\text { Age of PM } \\
\text { Implantation } \\
\text { (years/old) }\end{array}$ & UCG & Manifestation \\
\hline $\begin{array}{l}\text { III - } 10 \\
\text { Proband }\end{array}$ & M & 56 & 56 & $\begin{array}{l}\mathrm{LA}=40 \mathrm{~mm}, \mathrm{LV}=46 \mathrm{~mm}, \mathrm{IVS}=14 \mathrm{~mm}, \mathrm{LVPW}= \\
13 \mathrm{~mm}, \mathrm{EF}=65 \%\end{array}$ & $\begin{array}{l}\text { 3rd - degree } \\
\text { AVB,CRBBB,PVC }\end{array}$ \\
\hline II - 5 Uncle & M & 91 & 55 & $\begin{array}{l}\mathrm{LA}=38 \mathrm{~mm}, \mathrm{LV}=55 \mathrm{~mm}, \mathrm{RA}=37 \mathrm{~mm}, \mathrm{RV}= \\
37 \mathrm{~mm}, \mathrm{EF}=55 \%\end{array}$ & 3rd - degree AVB \\
\hline II - 7 Uncle & M & 89 & 50 & $\begin{array}{l}\mathrm{LA}=42 \mathrm{~mm}, \mathrm{LV}=62 \mathrm{~mm}, \mathrm{RA}=37 \mathrm{~mm}, \mathrm{RV}= \\
39 \mathrm{~mm}, \mathrm{EF}=40 \%\end{array}$ & 3rd - degree AVB,PVC \\
\hline II - 9 Father & $M$ & 86 & 59 & $\begin{array}{l}\mathrm{LA}=38 \mathrm{~mm}, \mathrm{LV}=50 \mathrm{~mm}, \mathrm{RA}=34 \mathrm{~mm}, \mathrm{RV}= \\
35 \mathrm{~mm}, \mathrm{EF}=54 \%\end{array}$ & 3rd - degree AVB,PVC \\
\hline $\begin{array}{l}\text { II }-11 \\
\text { Uncle }\end{array}$ & $M$ & 84 & 55 & Normal & 3rd - degree AVB \\
\hline $\begin{array}{l}\text { III }-12 \\
\text { Sister }\end{array}$ & $\mathrm{F}$ & 52 & - & Normal & None \\
\hline $\begin{array}{l}\text { IV - } 1 \\
\text { Daughter }\end{array}$ & $\mathrm{F}$ & 26 & - & Normal & None \\
\hline
\end{tabular}

\section{Genetic testing}

A nonsense mutation in TNNI3K(c.1441C > T,p.R481*), was found through WES and confirmed by Sanger sequencing(Fig. 1E). This variant produces a truncated protein with 481 amino acids which is significantly shorter than the wild-type protein (835 amino acids). It is a rare mutation with MAF of $0.0017 \%(2 / 119514$ alleles) in ExAC database.In addition, this mutation was not seen in our 200 local control cohorts[25]. The c.1441C > T mutation in the TNNI3K gene co-segregated with the clinical phenotype in this family.Thus,we believed that these mutation seem to be the causative mutation of cardiac abnormalities in this family.

\section{Discussion}

Herein,using NGS we have identified a nonsense mutation of the TNNI3K gene associated with CCD and DCM. Cardiovascular disease caused by the TNNI3K mutation is an autosomal dominant disease, which means individuals will have the disease if only one chromosome carries the mutant allele. In our study,the nonsense mutation(c.1441C > T) is co-segregated with the affected ones.

The gene TNNI3K holds a place in cardiac physiology. A preponderance of evidence suggested that TNNI3K is associated with a broad spectrum of cardiac phenotypes including CCD ,DCM,and supraventricular tachycardia. Only four disease-causing mutations(c.1577G > A,c.1615A > G,c.333 + 2T > C and c.2303G > A) in TNNI3K,namely,three missense mutations and one splicing mutation, have been reported so far(Fig. 1F). Among them,one locates in ANK repeats domain,one in serine-rich domain,and the rest two in kinase activity domain. Our study reported the first nonsense mutation that was linked with cardiovascular diseases, located in kinase activity domain. This mutation results in the early appearance of stop codon which is anticipated to produce truncated protein lacking partial protein kinase domain along with the C-terminus. For all we know, a premature termination codon (PTC) may result in loss of function (LOF) through activating a process called nonsense-mediated mRNA decay (NMD) [26, 27]. Indeed ,LOF is the mechanism for TNNI3K mutation leading to DCM and CCD proved by previous research. Therefore, we hold the opinion that this nonsense mutation is the likely pathogenic variant in the family. Truncated mutation have larger effects in protein function in comparison with other mutation types. Therefore, it is anticipated to have more serious phenotype than missense mutation in most instances. CCD of TNNI3K mutation carriers in our study seems to be more serious than previous reported cases based on the fact that all of them were highly PM-dependent. Cardiac enlargement was aggravated by time in follow-up period in our research. However, the extent of cardiac enlargement in our patients is not more serious than previous cases.Considering the oldest patient in 
this family is 93 , one would expect that loss of function in TNNI3K without detriment to life expectancy. In conclusion,more studies will need to be identified to draw clear genotype-phenotype relationships of TNNI3K.

However, there are several limitations in our research.This study lacks of direct evidence in elucidating the underlying pathways between TNNI3K and cardiac abnormalities. We could not confirm the stability of mutant TNNI3K mRNA/protein in the cells derived from affected members in the absence of the patients' consent.An animal model harboring specific mutation will be needed to elucidate the underlying signaling pathways.

\section{Conclusions}

we successfully identified a rare variant in TNNI3K(c.1441C $>$ T) that was associated with CCD and DCM. This mutation may affect the expression of TNNI3K through NMD. Altogether,we reported the first nonsense mutation associated with cardiovascular disease in this gene,paying the way for genetic diagnosis for CCD and DCM.

\section{Abbreviations}

ACMG

American College of Medical Genetics and Genomics guideline

ANK

ankyrin

CCD

cardiac conduction disease

CRBBB

complete right bundle branch block

DCM

dilated cardiomyopathy

ECG

electrocardiogram

HGMD

human gene mutation database

INDELS

insertion-deletion variants

IVS

interventricular septum

LA

left atrial

LOF

loss of function

LV

left ventricle

LVPW

left ventricular posterior wall

MAF

minor allele frequency

NGS

next generation sequencing

NMD

nonsense-mediated mRNA decay

PM

pacemaker

PTC 
premature termination codon

RA

right atrial

RV

right ventricle

SNVs

Single-nucleotide variants

UCG

ultrasonic cardiography

WES

whole exome sequencing

\section{Declarations}

\section{Ethics approval and consent to participate}

Written informed consent was obtained from each individual and the investigation was approved by the Ethics Committee of The Second Xiangya Hospital of

Central South University.

\section{Consent for publication}

All participants have provided informed written consent to genetic and biochemical analysis and to publication of all identifying images.

\section{Competing interests}

The authors declare that they have no conflict of interest.

\section{Availability of data and materials}

All data generated or analysed during this study are included in this published article and its supplementary information files.

Acknowledgements

Thank you for all study participants.

\section{Funding}

The whole course of this study including the study design, data collection, data analysis, data interpretation and manuscript preparation were mainly supported by National Natural Science Foundation of China (\#81570310 and \#81770337).

Author contributions

Q-M $L$ and $J L$ conceived the design and performed the study.JL and $D L$ analysed the data. JL wrote the original manuscript.Q-M $L, D L, K-K$ W,M-Z L,NL,C-Y Z and X-L S revised the the article. All authors reviewed and approved the final manuscript. 


\section{References}

1.

Baruteau AE, Probst V, Abriel H. Inherited progressive cardiac conduction disorders. CURR OPIN CARDIOL. 2015;30(1):33-9.

2.

Kaess BM, Andersson C, Duncan MS, Larson MG, Aasbjerg K, Gislason GH, Torp-Pedersen C, Vasan RS. Familial Clustering of Cardiac Conduction Defects and Pacemaker Insertion. Circ Arrhythm Electrophysiol. 2019;12(7):e7150.

3.

Brignole M, Auricchio A, Baron-Esquivias G, Bordachar P, Boriani G, Breithardt OA, Cleland J, Deharo JC, Delgado V, Elliott PM, et al. 2013 ESC Guidelines on cardiac pacing and cardiac resynchronization therapy: the Task Force on cardiac pacing and resynchronization therapy of the European Society of Cardiology (ESC). Developed in collaboration with the European Heart Rhythm Association (EHRA). EUR HEART J. 2013;34(29):2281-329.

4.

Yancy CW, Jessup M, Bozkurt B, Butler J, Casey DJ, Drazner MH, Fonarow GC, Geraci SA, Horwich T, Januzzi JL, et al. 2013 ACCF/AHA guideline for the management of heart failure: executive summary: a report of the American College of Cardiology Foundation/American Heart Association Task Force on practice guidelines. CIRCULATION. 2013;128(16):1810-52.

5.

Pinto YM, Elliott PM, Arbustini E, Adler Y, Anastasakis A, Bohm M, Duboc D, Gimeno J, de Groote P, Imazio M, et al. Proposal for a revised definition of dilated cardiomyopathy, hypokinetic non-dilated cardiomyopathy, and its implications for clinical practice: a position statement of the ESC working group on myocardial and pericardial diseases. EUR HEART J. 2016;37(23):1850-8.

6.

Dec GW, Fuster V. Idiopathic dilated cardiomyopathy. N Engl J Med. 1994;331(23):1564-75.

7.

McNally EM, Mestroni L. Dilated Cardiomyopathy: Genetic Determinants and Mechanisms. CIRC RES. 2017;121(7):731-48.

8.

Weintraub RG, Semsarian C, Macdonald P. Dilated cardiomyopathy. LANCET. 2017;390(10092):400-14.

9.

Zhao Y, Meng XM, Wei YJ, Zhao XW, Liu DQ, Cao HQ, Liew CC, Ding JF. Cloning and characterization of a novel cardiac-specific kinase that interacts specifically with cardiac troponin I. J Mol Med (Berl). 2003;81(5):297-304.

10.

Szabo L, Morey R, Palpant NJ, Wang PL, Afari N, Jiang C, Parast MM, Murry CE, Laurent LC, Salzman J. Statistically based splicing detection reveals neural enrichment and tissue-specific induction of circular RNA during human fetal development. GENOME BIOL. 2015;16:126.

11.

Feng Y, Liu DQ, Wang Z, Liu Z, Cao HQ, Wang LY, Shi N, Meng XM. AOP-1 interacts with cardiac-specific protein kinase TNNI3K and down-regulates its kinase activity. Biochemistry. 2007;72(11):1199-204.

12.

Wang H, Wang L, Song L, Zhang YW, Ye J, Xu RX, Shi N, Meng XM. TNNI3K is a novel mediator of myofilament function and phosphorylates cardiac troponin I. BRAZ J MED BIOL RES. 2013;46(2):128-37.

13.

Lodder EM, Scicluna BP, Milano A, Sun AY, Tang H, Remme CA, Moerland PD, Tanck MW, Pitt GS, Marchuk DA, et al. Dissection of a quantitative trait locus for PR interval duration identifies Tnni3k as a novel modulator of cardiac conduction. PLOS GENET. 2012;8(12):e1003113.

14.

Lai ZF, Chen YZ, Feng LP, Meng XM, Ding JF, Wang LY, Ye J, Li P, Cheng XS, Kitamoto Y, et al. Overexpression of TNNI3K, a cardiacspecific MAP kinase, promotes P19CL6-derived cardiac myogenesis and prevents myocardial infarction-induced injury. Am J Physiol Heart Circ Physiol. 2008;295(2):H708-16.

15.

Tang H, Xiao K, Mao L, Rockman HA, Marchuk DA. Overexpression of TNNI3K, a cardiac-specific MAPKKK, promotes cardiac dysfunction. J MOL CELL CARDIOL. 2013;54:101-11.

Page $7 / 9$ 
16.

Wang X, Wang J, Su M, Wang C, Chen J, Wang H, Song L, Zou Y, Zhang L, Zhang Y, et al. TNNI3K, a cardiac-specific kinase, promotes physiological cardiac hypertrophy in transgenic mice. PLOS ONE. 2013;8(3):e58570.

17.

Patterson M, Barske L, Van Handel B, Rau CD, Gan P, Sharma A, Parikh S, Denholtz M, Huang Y, Yamaguchi Y, et al. Frequency of mononuclear diploid cardiomyocytes underlies natural variation in heart regeneration. NAT GENET. 2017;49(9):1346-53.

18.

Wheeler FC, Tang H, Marks OA, Hadnott TN, Chu PL, Mao L, Rockman HA, Marchuk DA. Tnni3k modifies disease progression in murine models of cardiomyopathy. PLOS GENET. 2009;5(9):e1000647.

19.

Gan P, Patterson M, Velasquez A, Wang K, Tian D, Windle JJ, Tao G, Judge DP, Makita T, Park TJ, et al. Tnni3k alleles influence ventricular mononuclear diploid cardiomyocyte frequency. PLOS GENET. 2019;15(10):e1008354.

20.

Theis JL, Zimmermann MT, Larsen BT, Rybakova IN, Long PA, Evans JM, Middha S, de Andrade M, Moss RL, Wieben ED, et al. TNNI3K mutation in familial syndrome of conduction system disease, atrial tachyarrhythmia and dilated cardiomyopathy. HUM MOL GENET. 2014;23(21):5793-804.

21.

Xi Y, Honeywell C, Zhang D, Schwartzentruber J, Beaulieu CL, Tetreault M, Hartley T, Marton J, Vidal SM, Majewski J, et al. Whole exome sequencing identifies the TNNI3K gene as a cause of familial conduction system disease and congenital junctional ectopic tachycardia. INT J CARDIOL. 2015;185:114-6.

22.

Whole exome sequencing identifies a novel mutation (c.333 $+2 \mathrm{~T}>\mathrm{C})$ of TNNI3K in a Chinese family with dilated cardiomyopathy and cardiac conduction disease. GENE 2018, 648:63-67.

23.

Podliesna S, Delanne J, Miller L, Tester DJ, Uzunyan M, Yano S, Klerk M, Cannon BC, Khongphatthanayothin A, Laurent G, et al. Supraventricular tachycardias, conduction disease, and cardiomyopathy in 3 families with the same rare variant in TNNI3K (p.Glu768Lys). HEART RHYTHM. 2019;16(1):98-105.

24.

Richards S, Aziz N, Bale S, Bick D, Das S, Gastier-Foster J, Grody WW, Hegde M, Lyon E, Spector E, et al. Standards and guidelines for the interpretation of sequence variants: a joint consensus recommendation of the American College of Medical Genetics and Genomics and the Association for Molecular Pathology. GENET MED. 2015;17(5):405-24.

25.

Xiang R, Fan LL, Huang H, Cao BB, Li XP, Peng DQ, Xia K. A novel mutation of GATA4 (K319E) is responsible for familial atrial septal defect and pulmonary valve stenosis. GENE. 2014;534(2):320-3.

26.

Lykke-Andersen S, Jensen TH. Nonsense-mediated mRNA decay: an intricate machinery that shapes transcriptomes. Nat Rev Mol Cell Biol. 2015;16(11):665-77.

27.

Kurosaki T, Maquat LE. Nonsense-mediated mRNA decay in humans at a glance. J CELL SCl. 2016;129(3):461-7.

\section{Figures}



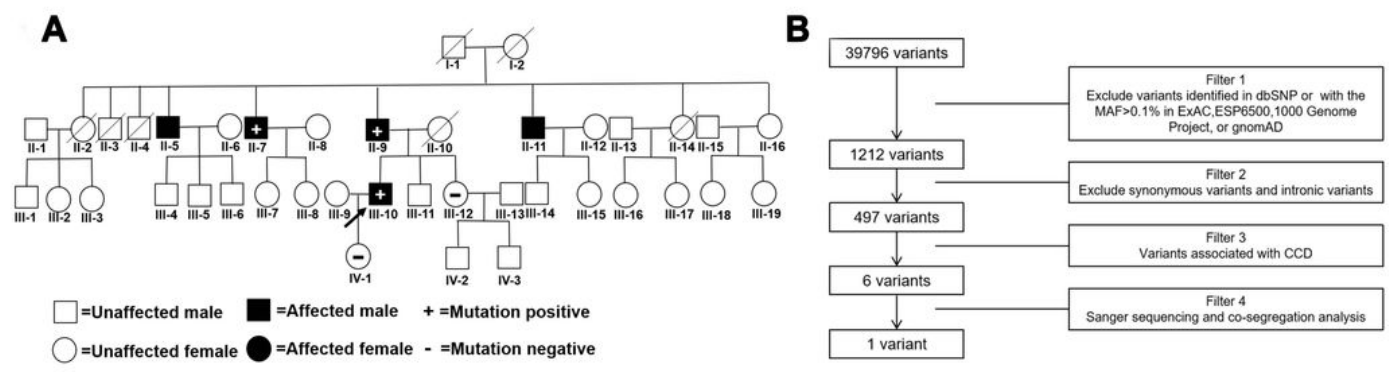

\section{C}

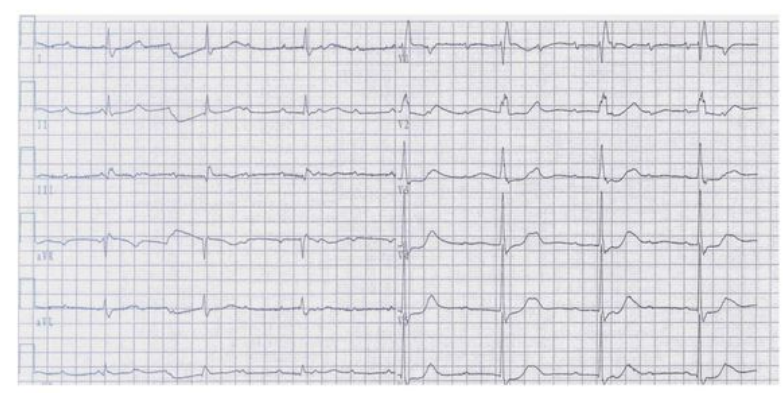

D

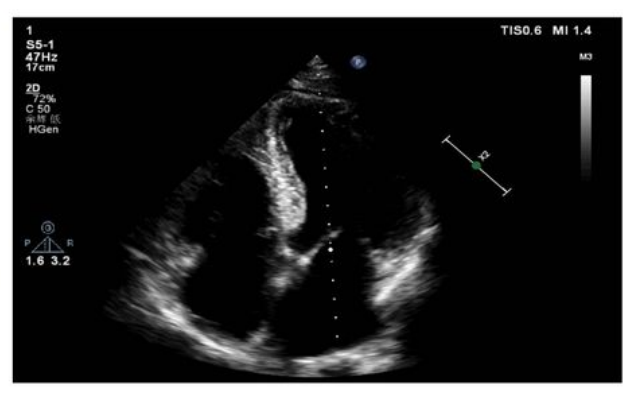

E

III-10

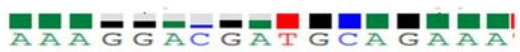

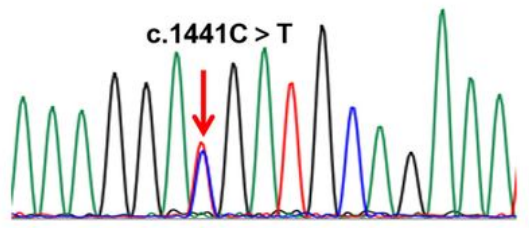

III-12

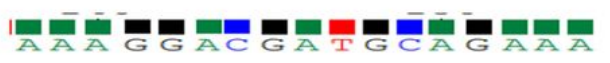

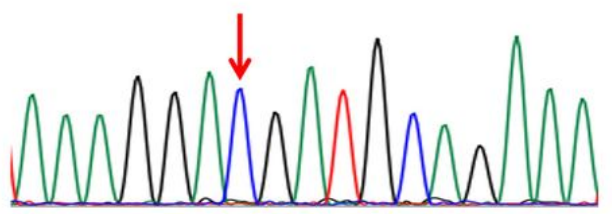

$\mathbf{F}$

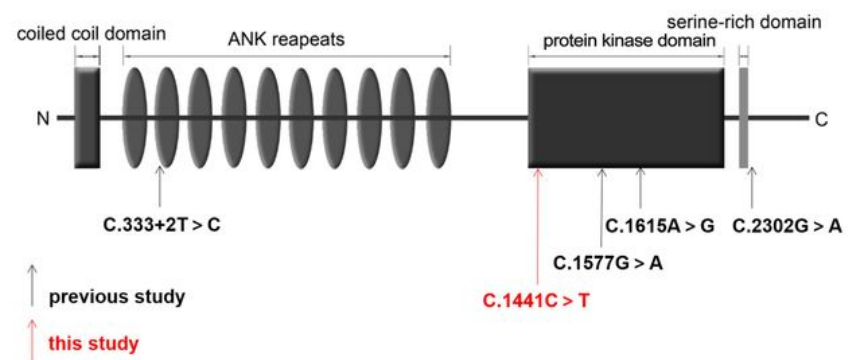

Figure 1

Pedigree, clinical data, sequencing data in our study. A:Pedigree.WES was performed on the proband(III-10). TNNI3K c.1441C>T variant was positive in individuals(II-7,II-9 and III-10) and negative in individuals(III-12 and IV-1). Other members' blood was not available. B:The ECG records of the proband(III-10) before PM implantation.C:The Echocardiography of III-10. D:Filter strategies in our research.E:Sanger sequencing confirmation.F:Mapping of the domain with TNNI3K variants identified in previous studies (denoted by black arrows) and this study (denoted by red arrow). NGS=next generation sequecing,WES=whole exome sequencing, $C C D=$ cardiac conduction disease $E C G=$ electrocardiogram, $P M=$ pacemaker. $A N K=$ ankyrin 